Bei dieser Untersuchung habe ich mich der geschickten Unter-

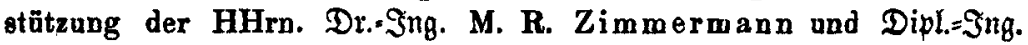
Hang Litter zu erfreuen gehabt.

Dresden, 11. Juni 1904.

402. M. Busoh: Ueber das Verhalten magnesiumorganischer Verbindungen zu Benzylidenanilin.

[Vorlāufige Mitth. aus dem chem. Institut der Univers. Erlangen.] (Eingegangen am 29. Juni 1904.)

Die in letzter Zeit mit so glänzendem Erfolge in die organische Synthese eingeführten magnesiumorganischen Verbindungen reagiren anch mit Alkylidenbasen, indem Addition an der Gruppe .HC:N. stattfindet; hierdurch ist ein Weg gegeben, ron den sogen. Schiff'schen Basen zu neuen secundären Aminen zu gelangen. So erbält man aus Benzylidenanilin und Magoesiumhalogenalkyl Additionsproducte, welche durch Wasser zu alkylirten Benzylanilinen zerlegt werden, die das Alkyl am Methankohlenstoff des Benzyls tragen. Die Vorgänge spielen sich folgendermaassen ab:

I. $\mathrm{C}_{6} \mathrm{H}_{3} \cdot \mathrm{N}: \mathrm{CH} \cdot \mathrm{C}_{6} \mathrm{H}_{5}+\mathrm{MgRJ}=\mathrm{C}_{6} \mathrm{H}_{5} \cdot \mathrm{N}-\mathrm{CH} \cdot \mathrm{C}_{6} \mathrm{H}_{5}$ Mg J $\dot{R}$

II. $\mathrm{C}_{6} \mathrm{H}_{5} \cdot \mathrm{N}-\mathrm{CH} . \mathrm{C}_{6} \mathrm{H}_{5}+\mathrm{H}_{2} \mathrm{O}=\mathrm{C}_{6} \mathrm{H}_{5} \cdot \mathrm{NH} . \mathrm{CHR} . \mathrm{C}_{6} \mathrm{H}_{5}+\mathrm{MgOHJ}$. Mg J $\dot{R}$

In Gemeinschaft mit Hrn. A. Rinck babe ich rorerst das Verbalten des Benzylidenanilins gegen Methyl- und Phenyl-Magnesiumjodid studirt; wir hoffen demnächst weitere Mittheilungen über diese nene Verwendung der magnesiumorganischen Verbindungen machen zu können.

$$
\begin{gathered}
C \text {-Methylbenzyl-anilin, } \mathrm{C}_{6} \mathrm{H}_{6} . \mathrm{NH} . \mathrm{CH}\left(\mathrm{CH}_{3}\right) \cdot \mathrm{C}_{6} \mathrm{H}_{3} \text {. } \\
\text { ( } \alpha \text {-Anilidoäthyl-benzol.) }
\end{gathered}
$$

Benzylidenanilin wird in äquimolekularer Menge zu der in bekannter Weise bereiteten ätherischen Lösung von Methylmagnesiumjodid gegeben, der Aether auf dem Wasserbade vollkommen abdestillirt und die rückstãndige braune, syrupöse Masse mit Wasser zersetzt. Dem resultirenden dick füssigen Oel kann der basische Antheil durch Erwärmen mit verdūnnten Mineralsăuren entzogen werden; zweckmässig verwendet man hierzu Salpetersäure, die mit dem entstandenen Methylbenzyl-anilin ein in kaltem Wasser schwer lösliches 
Salz bildet. Man säuert die wässrige Flüssigkeit mit verdünnter Salpetersäure an, kocht auf und filtrirt von der ungelöst bleibenden grünen, schmierigen Masse ab. Beim Erkalten fällt das Nitrat der Base in glänzenden Nadeln an. Das Rohproduct erscheint durch das erwähnte Nebenproduct noch grünlich gefärbt und erwies sich ausserdem als etwas jodhaltig durch beigemengtes Jodhydrat. Durch Umkrystallisiren aus mit Salpetersäure angesäuertem Wasser gewinnt man das Salz in schönen, weissen, glänzenden Nadeln, die bei 162- $163^{0}$ schmelzen und sich leicht in Alkohol lösen. Die Ausbente ist gut, aus $15 \mathrm{~g}$ Benzylidenanilin gewannen wir $17 \mathrm{~g}$ Salz.

Das Methylbenzyl-anilin stellt ein dickflüssiges, in reinem Zustande farb- und fast geruch-loses Oel dar, das bisher nicht zum Erstarren gebracht wurde; es siedet unter $20 \mathrm{~mm}$ Druck bei $183^{\circ}$.

Zur Analyse haben wir das Nitrat verwandt.

$0.2019 \mathrm{~g}$ Sbst.: $0.4761 \mathrm{~g} \mathrm{CO}, 0.1161 \mathrm{~g} \mathrm{H}_{2} 0 .-0.1996 \mathrm{~g}$ Sbst.: $20.1 \mathrm{ccm} \mathrm{N}$ $\left(28^{0}, 743 \mathrm{~mm}\right)$.

$$
\begin{aligned}
& \mathrm{C}_{14} \mathrm{H}_{15} \mathrm{~N} . \mathrm{HNO}_{3} \text {. Ber. C 64.61, H 6.15, N 10.77. } \\
& \text { Gef. 》 64.31. * 6.44, 》 } 10.86 \text {. }
\end{aligned}
$$

Das Chlorhydrat, $\mathrm{C}_{14} \mathrm{H}_{15} \mathrm{~N} . \mathrm{HCl}$, schiesst beim Lösen der Base in verdünnter Salzsäure bald in weissen Nadeln an, die in Wasser ziemlich leicht löslich sind, aus dieser Lösung aber durch concentrirte Säure leicht wieder gefällt werden kōnnen. Leicht löslich in Alkohol. Schmp. 184-1850.

0.233 g Sbst.: $0.1407 \mathrm{~g} \mathrm{Ag} \mathrm{Cl} .-0.2567$ g Sbst.: $14.4 \mathrm{ccm} \mathrm{N}\left(24^{0}, 736 \mathrm{~mm}\right)$.

$$
\begin{aligned}
& \mathrm{C}_{14} \mathrm{H}_{15} \mathrm{~N} \text {. HCl. Ber. Cl 15.20, N 5.99. } \\
& \text { Gef. 》 14.94, } 6.08 \text {. }
\end{aligned}
$$

Besonders schön krystallisirt das Sulfat $\left(\mathrm{C}_{14} \mathrm{H}_{15} \mathrm{~N}\right)_{2} \cdot \mathrm{H}_{2} \mathrm{SO}_{4}$. Während die concentrirte, warme Lösung der Base in verdünnter Schwefelsäure weisse Nadeln fallen lässt, erhält man aus verdünnteren Lösungen gut ausgebildete, sechsseitig begrenzte Täfelchen des monoklinen Systems. Schmp. $142-143^{\circ}$. Gleich den übrigen Salzen erleidet auch das Sulfat in Wasser einen partiellen Zerfall in Base und Säure.

$0.2094 \mathrm{~g}$ Sbst.: $0.0964 \mathrm{~g} \mathrm{BaSO}_{4}$.

$$
\left(\mathrm{C}_{44} \mathrm{H}_{15} \mathrm{~N}\right)_{2} . \mathrm{H}_{2} \mathrm{SO}_{4} \text {. Ber. } \mathrm{H}_{2} \mathrm{SO}_{4} \text { 19.92. Gef. } \mathrm{H}_{2} \mathrm{SO}_{4} 19.55 \text {. }
$$

Versetzt man die Lösung eines der Salze mit Natriumnitrit, so fällt das Nitrosamin, $\mathrm{C}_{6} \mathrm{H}_{3} \cdot \mathrm{N}(\mathrm{NO}) \cdot \mathrm{CH}\left(\mathrm{CH}_{3}\right) \cdot \mathrm{C}_{6} \mathrm{H}_{5}$, als bräunlichgelbes Oel aus, das die Liebermann'sche Reaction giebt. Es kann in normaler Weise in die Nitroso-Verbindung umgelagert werden. Fügt man zar ätherischen Lösung des Nitrosamins alkobolische Salzsäure, so scheidet sich bald ein Salz in Nadeln ab, aus dem durch Alkali die Nitrosobase als grünes Oel in Freiheit gesetzt wird. Beim 
Lösen des Balzes in Wasser tritt Geruch nach Acetophenon auf, der auch beim Verarbeiten der Salze des Methylbenzyl-anilins stets bemerkbar ist. Die Bildung von Acetophenon ist natürlich auf dieselbe Ursache zurückzuführen, wie das von 0 . Fischer ${ }^{1}$ ) beobachtete Auftreten von Benzaldehyd bezw. Benzylidenanilinchlorhydrat bei der Umlagerung des Phenylbenzylnitrosamins. Unter dem Einfluss der salpetrigen Säure wird das Methylbenzyl-anilin oxydirt:

$$
\mathrm{C}_{6} \mathrm{H}_{5} \cdot \mathrm{NH} \cdot \mathrm{CH}\left(\mathrm{CH}_{8}\right) \cdot \mathrm{C}_{6} \mathrm{H}_{5}+\mathrm{O}=\mathrm{C}_{6} \mathrm{H}_{5} \cdot \mathrm{N}: \mathrm{C}_{\left(\mathrm{CH}_{8}\right)} \cdot \mathrm{C}_{6} \mathrm{H}_{5}+\mathrm{H}_{2} \mathrm{O} \text {, }
$$
worauf das Oxydationsproduct durch die anwesende Săure sofort der hydrolytischen Spaltung anheimfällt

$$
\mathrm{C}_{6} \mathrm{H}_{6} \cdot \mathrm{N}: \mathrm{C}\left(\mathrm{CH}_{3}\right) \cdot \mathrm{C}_{6} \mathrm{H}_{6}+\mathrm{H}_{2} \mathrm{O}=\mathrm{C}_{6} \mathrm{H}_{5} \cdot \mathrm{NH}_{2}+\mathrm{C}_{6} \mathrm{H}_{5} \cdot \mathrm{CO} \cdot \mathrm{CH}_{3} \text {. }
$$

Es wird zu prüfen sein, wie weit dieser Process, der eine Synthese ron Ketonen aus Aldehyden in sich schliesst, praktisch durchführbar ist.

Mit Phenylcyanat vereinigt sich unser Methylbenzyl-anilin zum

$$
\begin{gathered}
a \cdot \text { Phenyl- } b \text {-phenyl-b-phenylätbyl-harnstoff, } \\
\mathrm{C}_{6} \mathrm{H}_{6} \cdot \mathrm{N} \cdot \mathrm{CH}\left(\mathrm{C}_{6} \mathrm{H}_{5}\right) \cdot \mathrm{CH}_{3} \\
\text { CO.NH. } \mathrm{C}_{6} \mathrm{H}_{5} .
\end{gathered}
$$

Die Reaction erfolgt erst bei Wasserbad-Temperatur und erfordert einige Zeit zur Vollendung. Das dickflüssige Reactionsproduct wurde in wenig Aether gelöst und Gasolin hinzugefügt, worauf der Harnstoff sich in glänzenden, derben Krystallen absetzte. Bei langsamem Verdunsten der Lösung in Aether-Gasolin erhält man prächtige, vierseitige, rechtwinklig begrenzte, wasserhelle Tafeln. Aus verdūnntem Alkohol fielen derbe Blätter an. Der Körper schmilzt bei $94-95^{\circ}$; er ist leicht löslich in Aether, Benzol und Alkohol, schwer in Gasolin.

\section{$0.1494 \mathrm{~g}$ Sbst. $12.2 \mathrm{ccm} \mathrm{N}\left(22^{\circ}, 742 \mathrm{~mm}\right)$.}

$$
\mathrm{C}_{31} \mathrm{H}_{20} \mathrm{ON}_{2} \text {. Ber. N 8.86. Gef. N } 9.03 \text {. }
$$

$$
\text { Diphenyl-anilido-methan, } \mathrm{C}_{6} \mathrm{H}_{6} \cdot \mathrm{NH} \text {. CH }\left(\mathrm{C}_{6} \mathrm{H}_{5}\right)_{2} \text {, }
$$

ans Benzylidenanilin und Phenylmagnesiumjodid. Die ätherische Lösung der Componenten wird auf dem Wasserbad abdestillirt, das als zähe Masse zurückbleibende Additionsproduct mittels Wasser zerlegt and das resultirende Oel nach Zugabe von etwas Ammoniak und Salmiak mit Aether aufgenommen. Der ätherische Auszug lieferte auf Zusatz von alkoholischer Salzsäure das

Chlorhydrat des Diphenylanilidomethans in glänzenden, grünlichen Nadeln, die durch Umkrystallisiren aus Alkohol fast farblos

1) Ann. d. Chem. 241, 331 . 
und analymenrein erhalten wurden. Das Salz ist ziemlich schwer löslich in absolutem Alkohol, fast unlöslich in Wasser, von dem es beim Erwärmen vollkommen zerlegt wird. Schmp. $199^{\circ}$.

0.2325 g Sbst.: $10.2 \mathrm{ccm} \mathrm{N}\left(23^{\circ}, 740 \mathrm{~mm}\right)$.

$\mathrm{C}_{19} \mathrm{H}_{17} \mathrm{~N}$. HCl. Ber. N 4.74. Gef. N 4.81.

Die Base wurde vorerst als sehr dickflüssiges Oel erhalten, das noch der näheren Untersuchung harrt.

403. H. Erdmann und O. Makowka: Die Bestimmung des Palladiums und dessen Trennung von anderen Metallen durch Acetylen.

[Vorlanfige Mitth. a. d. Anorg.-Chem. Lab. der Kgl. Techn. Hochsch. zu Berlin.] (Eingegangen am 17. Juni 1904.)

Eine soeben erschienene Veröffentlichung von Jannasch und Bettges ${ }^{1)}$ veranlasst uns, aus einer grösseren Arbeit über die Verwendung des Acetylens in der qualitativen und quantitativen Analyse, welche wir demnächst abschliessen zu können hoffen, bereits heute eine karze, speciell das Palladium betreffende Mittheilung zu machen.

So sehr wir jedes neue analytische Reagens mit Freude begrüssen und namentlich die Einführung des Hydrazins in die quantitative Analyse, wie sie durch die schönen Arbeiten von Jannasch und seinen Mitarbeitern vorgeschlagen und begründet worden ist, für eine überaus glückliche und fruchtbringende halten, so glauben wir doch nicht, dass dieses Reagens speciell für die Abscheidung and Bestimmung des Palladiums besondere Vorzüge besitzt, welche seine allgemeine Anwendung empfehlenswerth machen. Denn für jedes Metall suchen wir doch in erster Linie nach einem charakteristischen Fällungs- und Scheidungs-Mittel; ein solches ist aber das Hydrazin für das Palladium offenbar nicht. Das Hydrazin wirkt bei dem Jannasch'schen Verfabren in saurer Lösung nur dorch seine Reductionskraft, das Palladium ist aber nicht etwa wie das Rhodium oder auch das Gold vor seinen Begleitmetallen durch eine besonders leichte Reducirbarkeit ausgezeichnet. Daher stiessen Jannasch und Bettges bereits auf Schwierigkeiten, als sie ein kupferhaltiges Palladium in der angegebenen Weise analysirten. Der Niederschlag schloss bis zu anderthalb Procent Kupfer ein, und die Operation musste, damit man ein

1) Die Bestimmung des Palladiums und dessen Trennung ron anderen Metallen durch Hydrazin, diese Berichte 37, 2210 [1904]. 